'Departamento de Matemática y Estadística, Facultad de Ciencias Bioquímicas y Farmacéuticas, Universidad Nacional de Rosario. Rosario, Argentina.

aioquímica, Farmacéutica. bIngeniero Químico.

'Alumna de la carrera de Licenciatura en Química.

dAlumno de la carrera de Licenciatura en Biotecnología. eProfesora de Matemática. fLicenciada en Biotecnología.

Fuentes de apoyo financiero: El proyecto que dio origen a este trabajo no tuvo apoyo financiero.

Recibido el 11 de abril de 2014 aceptado el 27 de noviembre de 2014.

Correspondencia a: Marta Mónica Marzi Presidente Julio Roca, N 653 Piso 7, Dpto. A. Rosario, Santa Fe, Argentina. Teléfonos: 03414253300 0341156560483 marzimarta58@gmail.com

\section{Efectos adversos cardíacos de la domperidona en pacientes adultos: revisión sistemática}

\author{
MARTA MARZI ${ }^{1, \mathrm{a}}$, DARÍO WEITZ ${ }^{1, \mathrm{~b}}$, AYLÉN AVILA $^{1, \mathrm{c}}$, \\ GABRIEL MOLINA $^{1, \mathrm{~d}}$, LUCÍA CARABALLO ${ }^{1, \mathrm{e}}$, LAURA PISKULIC ${ }^{1, \mathrm{f}}$
}

\section{Cardiac adverse effects of domperidone in adult patients: a systematic review}

Background: Domperidone is widely prescribed in patients with gastrointestinal disorders but some cardiac adverse effects have been recently reported. Aim: To evaluate the risk of QT prolongation, ventricular arrhythmias and sudden cardiac death associated with the use of oral domperidone in adults without cancer. Material and Methods: Systematic searches in MEDLINE, LILACS, SciELO, the Cochrane Library and regulatory agencies websites were performed, followed by a manual search of cited references. The search strategy consisted of combining free and indexed text words without any date or language restriction. Results: Three case-control studies met the inclusion criteria; none of them evaluated QT interval prolongation. With low risk of bias, each study quantified the risk of ventricular arrhythmia or sudden cardiac death (VA/ $S C D)$. The odds ratios for these events in these studies were 4.7 (95\% confidence interval (CI): 1.4-16), 1.59 (95\% CI: 1.28-1.98) and 11.02 (95\% CI: 2.02-62.3) respectively. A significantly increased risk was observed in patients older than 60 years of age or receiving doses $>30 \mathrm{mg} /$ day. Conclusions: Heterogeneity between selected studies did not allow the computation of a summary measure. However, evidence was found that an increased risk of VA/SCD is associated with the use of oral domperidone in adults.

(Rev Med Chile 2015; 143: 14-21)

Key words: Arrhythmias, cardiac; Domperidone; Long QT; Review.
L a domperidona es un agente procinético utilizado para el tratamiento de desórdenes tales como reflujo gastroesofágico, dispepsia, constipación y otros problemas de motilidad gastrointestinal. Reduce el tiempo de reflujo postprandial, motivo por el cual se la utiliza para tratar vómitos y regurgitación. Domperidona es un derivado benzoimidazólico antagonista periférico de los receptores dopaminérgicos $\mathrm{D}_{2}$; a diferencia de la metoclopramida, cruza con dificultad la barrera hematoencefálica por lo cual se han reportado pocos casos de efectos adversos en el sistema nervioso central ${ }^{1}$.

Se encuentra en el mercado farmacéutico desde 1978 reemplazando a otras drogas procinéticas tales como cisaprida y metoclopramida. Cisaprida fue retirada del mercado norteamericano debido a numerosos casos reportados de arritmia y prolongación del intervalo QT y sólo se la utiliza en algunos países bajo la recomendación de realizar un monitoreo periódico de la función cardíaca² Durante la década del 80 se administraba domperidona vía intravenosa para el control del vómito inducido por la quimioterapia citotóxica. Diversos 
reportes sobre arritmias fatales y otros incidentes cardiotóxicos tuvieron como consecuencia la recomendación de analizar cuidadosamente la aplicación de la variante intravenosa ${ }^{3}$.

A partir de la utilización creciente de domperidona en forma oral, se ha reportado prolongación del intervalo QT, arritmia ventricular e inclusive muerte súbita en adultos tras su administración ${ }^{4-6}$. Diversas agencias sanitarias han advertido acerca del uso de domperidona en cualquiera de sus presentaciones $^{7-14}$. En Estados Unidos la Food and Drug Administration (FDA) prohibió la administración de la droga ${ }^{15}$.

En Argentina se considera medicamento esencial para el tratamiento de náuseas y vómitos, tanto en su forma oral como inyectable. Está incluida en el Formulario Terapéutico donde se hace mención a los casos publicados de prolongación del intervalo QT y de Torsades de Pointes asociados al uso de domperidona, pero al no existir pruebas concluyentes sólo recomienda evitar la administración intravenosa y no usarla en mujeres embarazadas en ninguna de sus presentaciones ${ }^{16,17}$.

El objetivo de la presente revisión sistemática fue evaluar el riesgo de prolongación del intervalo QT, arritmia ventricular y muerte súbita cardíaca asociado al uso de domperidona administrada en forma oral para el tratamiento y prevención de náuseas, vómitos y reflujo gastroesofágico en la población adulta sin cáncer.

\section{Métodos}

\section{Criterios de elegibilidad}

Estudios epidemiológicos tanto experimentales como observacionales que evaluaron los efectos adversos cardíacos de la domperidona administrada en forma oral para el tratamiento y prevención de náuseas, vómitos y reflujo gastroesofágico en la población adulta sin cáncer.

\section{Estrategia de búsqueda para la identificación de los estudios}

Se consultaron las bases de datos electrónicas MEDLINE, LILACS, SciELO, la Biblioteca Cochrane, y los alertas y boletines de seguridad de los entes regulatorios nacionales e internacionales disponibles en línea, sin límite de fecha ni de idioma. La estrategia de búsqueda consistió en la combinación de términos indexados y de términos libres mediante la utilización de operadores booleanos [Ejemplo: Domperidone AND (arrhythmia OR QT OR death OR fibrillation OR torsades de pointes)]. Se especificó que la información requerida estuviera en el título y/o resumen y se la limitó a estudios en seres humanos. La búsqueda que se realizó en el período febrero-marzo de 2013, se complementó mediante la revisión de citas incluidas en los artículos completos recuperados y a través del contacto con centros y redes de investigación médica y farmacológica.

\section{Identificación y selección de los estudios}

Los artículos fueron identificados por tres autores según la estrategia descripta. La selección fue realizada por dos de ellos de manera independiente y los desacuerdos fueron resueltos por un tercer autor. En primer lugar se evaluó el título y el resumen de los artículos, en segundo lugar se recuperó el texto completo de los estudios potencialmente relevantes para ser sometidos nuevamente a evaluación para su elegibilidad.

\section{Extracción de los datos}

Se diseñó un formulario de extracción de datos específicos para esta revisión, donde se registró el diseño del estudio, la duración del mismo, las características de los pacientes, la intervención/ exposición y los resultados.

\section{Evaluación del riego de sesgo}

Se elaboró una herramienta para la evaluación del riesgo de sesgo de los estudios de casos y controles, con los siguientes ítems: descripción de la fuente de datos, definición del evento, identificación y verificación de los casos, cegamiento de la exposición en la selección de los casos, selección de los controles, descripción temporal de la exposición (reciente/pasada), identificación y análisis de los factores de confusión. A cada ítem cuatro autores en forma independiente le asignaron una valoración en relación al riesgo de sesgo: bajo, alto, incierto. Si el ítem fue informado en forma completa y el requisito implícito en él se cumplió satisfactoriamente, la valoración asignada fue "bajo riesgo de sesgo". Si el ítem fue informado en forma completa y el requisito se cumplió en forma poco satisfactoria o no se cumplió, la valoración asignada fue "alto riesgo de sesgo". Si no se proporcionó información, el riesgo se valoró como "incierto" 18 . 


\section{Resultados}

\section{Selección de los estudios}

La búsqueda en las bases de datos electrónicas consultadas produjo 66 títulos y resúmenes. Se excluyeron 43 artículos por no satisfacer criterios de elegibilidad (inclusión/exclusión). Se recuperaron los textos completos de los 23 remanentes. A partir de la lectura de los mismos se incorporaron 7 potenciales trabajos, dando un total de 30 artículos para una reevaluación detallada. Por los motivos indicados en la Figura 1, se excluyeron 27 artículos quedando un total de tres estudios ${ }^{19-21}$.

Un tercer revisor debió intervenir debido a desacuerdos en la inclusión/exclusión de un estudio. El ensayo clínico realizado por Boyce et $\mathrm{al}^{22}$, estudió prolongación del intervalo QT en voluntarios jóvenes sanos mientras que los tres artículos finalmente incluidos estudiaron arritmia ventricular y muerte súbita cardíaca con un diseño observacional de casos y controles. Se decidió excluir el trabajo de Boyce et al, debido a hetero- geneidad en diseño, población y evento estudiado.

Aunque sin desacuerdo entre los revisores, otro estudio relevante no se incluyó en la presente revisión. Strauss et $\mathrm{al}^{5}$, realizaron un estudio epidemiológico de casos y controles en Holanda para evaluar el riesgo de muerte súbita cardíaca asociado al uso de agentes no-cardíacos que prolongan el intervalo QT, entre ellos domperidona. Debido a que se identificó y seleccionó otro estudio de características similares focalizado en la domperidona $^{21}$ y que utilizó los registros de la misma base de datos durante un período de tiempo más prolongado, se decidió no incluir el estudio de Strauss et al, en la presente revisión.

\section{Evaluación del riesgo de sesgo}

El único ítem que recibió la categorización de "riesgo incierto" en dos de los trabajos ${ }^{19,20}, \mathrm{y}$ con acuerdo de los revisores, fue "cegamiento de la exposición en la selección de los casos". Los requisitos restantes fueron respondidos satisfactoriamente en los tres estudios.

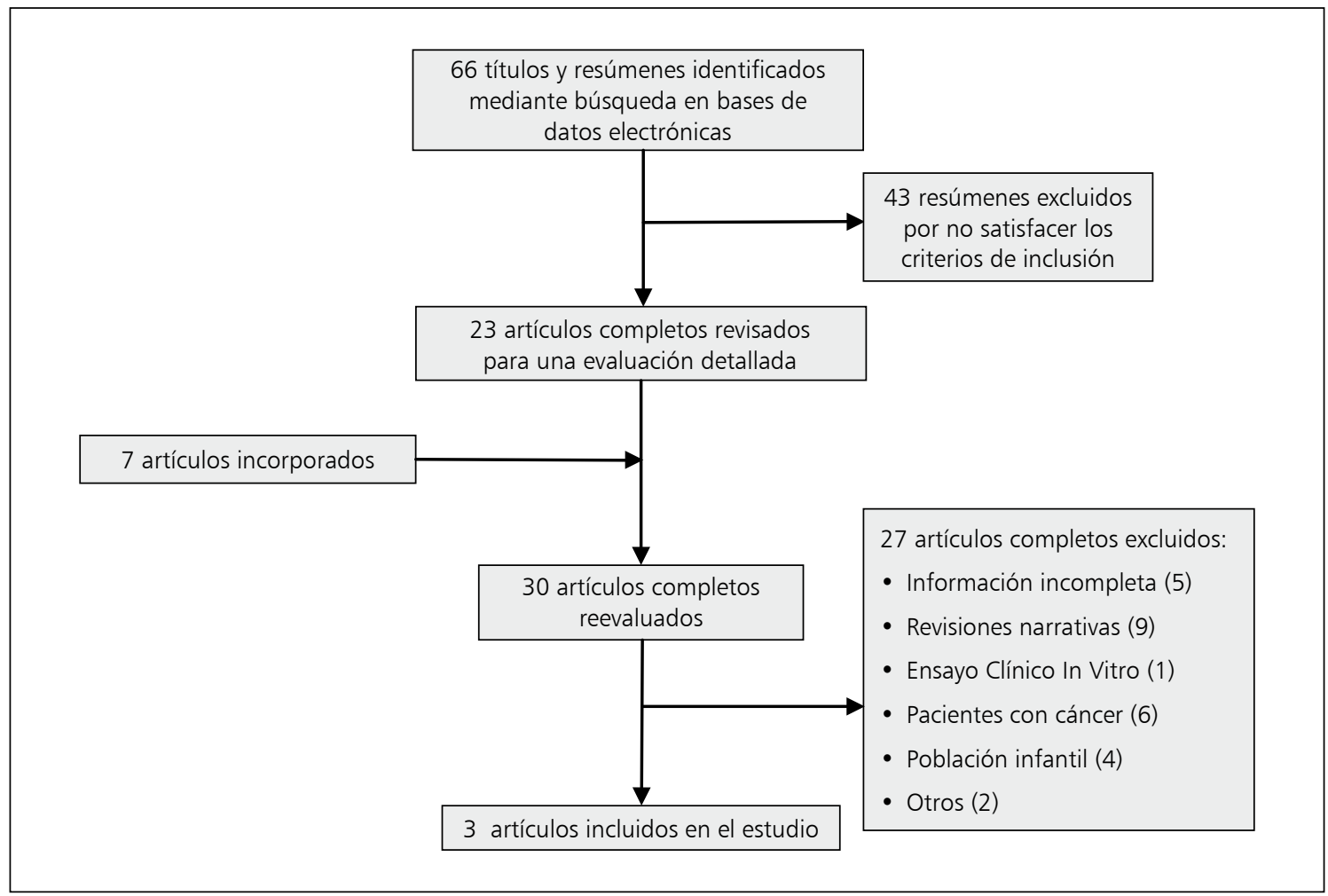

Figura 1. Identificación y selección de los estudios. 


\section{Características de los estudios incluidos}

Los tres estudios incluidos respondieron a un diseño de casos y controles. Las características de los mismos se detallan en la Tabla 1.

\section{Resultados de los estudios individuales}

Los tres estudios revelaron asociación estadísticamente significativa entre uso corriente de domperidona oral y arritmia ventricular severa y/o muerte súbita cardíaca (Tabla 2). En cambio, el uso pasado de la droga no se asoció con el evento de interés.

\section{De Bruin et al, 2006}

Los autores cuantificaron el riesgo de paro cardíaco asociado con el uso de drogas no anti-

Tabla 1. Característica de los estudios incluidos

\section{De Bruin et al, 2006}

\section{Métodos}

- Diseño: Estudio de casos y controles

- Fuente: Base de datos electrónica hospitalaria de pacientes admitidos en el Academic Medical Centre, Ámsterdam Holanda

- Período de estudio: Desde el 1 de enero de 1995 al 25 de diciembre de 2003

Participantes

- Casos: 140 pacientes que experimentaron paro cardíaco y necesitaron intervención del equipo de resucitación. Edad media de 59,6 años, 65,7\% del sexo masculino

- Controles: Pacientes hospitalizados en la fecha que ocurrió el evento. Cuatro controles por cada caso (560). Edad media de 47,5 años, $48,9 \%$ del sexo masculino

Exposición

Domperidona oral. Usuario corriente: evento ocurrido durante el período de prescripción de la droga; no usuario

\section{Johannes et al, 2010}

Métodos

- Diseño: Estudio de casos y controles, anidado

- Fuente: Base de datos electrónica Saskatchewan Health, Canadá

- Período de estudio: Desde el 1 de enero de 1990 al 31 de diciembre de 2005

Participantes

Rango de edad: 20-95 años. Edad media de 79, 4 años y el 47\% del sexo masculino

- Casos: 1.608 pacientes con muerte súbita cardíaca o arritmia ventricular severa

- Controles: Hasta 4 controles por cada caso (6.428)

Exposición

Domperidona oral. Usuario corriente: dentro de los 37 días previos al evento; usuario pasado: desde los 37 días previos y hasta un año; no expuestos: sin prescripción durante los 365 días previos

\section{Van Noord et al, 2010}

Métodos

- Diseño: Estudio de casos y controles

- Fuente: Base de datos electrónica de médicos de prácticas generales, Integral Primmary Care Informarion (IPCI), Holanda.

- Período de estudio: Desde el 1 de enero de 1995 al 1 de mayo de 2007

Participantes

Mayores de 18 años registrados con al menos un año completo de historia clínica

- Casos: 62 pacientes con arritmia ventricular no fatal y 1.304 correspondientes a muerte súbita cardíaca. Edad media de 72,5 años, el $58 \%$ del sexo masculino

- Controles: Hasta 20 controles por caso para muerte súbita (13.480) y hasta 40 controles por caso para arritmia ventricular (634)

Exposición

Domperidona oral. Usuario corriente: máximo de siete días desde la última prescripción hasta la ocurrencia del evento; usuario pasado: más de siete días desde la última prescripción; no expuesto: sin prescripción de la droga 
Tabla 2. Riesgo de efectos adversos cardíacos de la domperidona administrada en forma oral en pacientes adultos. Resultados de los estudios individuales

\begin{tabular}{|c|c|c|c|c|c|}
\hline Estudio/Autor, año & Evento & Casos & Controles & $\begin{array}{c}\text { Dosis } \\
\text { (mg/día) }\end{array}$ & $\begin{array}{l}\text { OR* ajustado } \\
\quad(95 \% \text { IC) }\end{array}$ \\
\hline De Bruin et al, 2006 & Paro cardíaco & $\begin{array}{c}(n=140) \\
7\end{array}$ & $\begin{array}{c}(n=560) \\
15\end{array}$ & - & $4,7 \quad(1,4-16)^{\mathrm{a}}$ \\
\hline Johannes et al, 2010 & $\begin{array}{l}\text { Muerte súbita cardíaca/arritmia } \\
\text { ventricular severa }\end{array}$ & $\begin{array}{c}(n=1.608) \\
169\end{array}$ & $\begin{array}{c}(n=6.428) \\
481\end{array}$ & - & $1,59(1,28-1,98)^{b}$ \\
\hline \multirow[t]{3}{*}{ Van Noord et al, 2010} & \multirow{3}{*}{$\begin{array}{l}\text { Muerte súbita cardíaca/arrritmia } \\
\text { ventricular }\end{array}$} & $(n=1.366)$ & $(n=14.114)$ & & \\
\hline & & 6 & 27 & $\leq 30$ & $1,36(0,37-5,04)^{c}$ \\
\hline & & 4 & 3 & $>30$ & $11,02(2,02-62,3)^{c}$ \\
\hline
\end{tabular}

*Razón de Odds. ajustado por edad, sexo, arritmias cardíacas, otras enfermedades cardíacas, diabetes mellitus, enfermedades pulmonares, número total de drogas utilizadas, uso concomitante de drogas antiarrítmicas, potasio sérico, calcio, magnesio, creatinina y bilirrubina. 'bjustado por condición médica, exposición corriente a medicamentos (drogas que prolongan el intervalo QT clase 1 y/o 2, inhibidores del CYP3A4) e indicadores de cuidados de la salud. 'Ajustado por insuficiencia cardíaca, tipo de seguro médico, inhibidores del CYP3A4, drogas inhibidoras de hERG, laxantes, digoxina, diuréticos, corticosteroides, agonistas beta-adrenérgicos.

arrítmicas conocidas por producir prolongación del intervalo QT. Mostraron que la razón de odds (OR) ajustada por potenciales factores de confusión dependía de la dosis, siendo 1,9 (95\% IC: $1,1-3,5)$ para dosis menores a 1 DDD (Dosis Diaria Definida) y 2,5 (95\% IC: 1,1-5,9) para dosis mayores. Encontraron además que el uso concomitante de dos o más drogas que prolongan el intervalo QT incrementaba el valor ajustado de OR a 4,8 (95\% IC: 1,6-14).

Dado que el estudio no fue diseñado para estudiar específicamente el riesgo asociado a cada droga en forma individual, al informar la razón de odds ajustada para domperidona no especificaron la dosis utilizada ni la edad de los pacientes (Tabla 2).

\section{Johannes et al, 2010}

Evaluaron el riesgo combinado de arritmia ventricular severa y muerte súbita cardíaca en pacientes que usaban domperidona y/o agentes inhibidores de la bomba de protones. En la Tabla 2 se muestra la razón de odds ajustada para los expuestos a domperidona respecto de los no expuestos a ninguna de las drogas estudiadas. No informaron la dosis oral administrada $y$, al igual que en los trabajos anteriores, los hallazgos estadísticamente significativos corresponden al uso corriente de la droga.

Los autores hicieron un análisis estratificado para determinar potenciales modificadores del efecto tales como edad, sexo y presencia o no de diabetes. La razón de odds ajustada para exposición corriente a domperidona comparada con no uso fue más alta y con significación estadística en los pares caso-control sin diabetes (OR: 1,69; 95\% IC: $1,32-2,17)$ que con diabetes (OR: 1,$27 ; 95 \%$ IC: $0,79-2,03$ ), en los mayores de 60 años (OR: 1,64; 95\% IC: $1,31-2,05)$ que en los individuos más jóvenes (OR: 1,10; 95\% IC: 0,35-3,47) y en hombres (OR: 2,23; 95\% IC: 1,59-3,13) que en mujeres (OR: 1,25; 95\% IC: 0,93-1,67).

\section{Van Noord et al, 2010}

Este estudio mostró que sólo el uso corriente de domperidona oral en dosis superiores a $30 \mathrm{mg} /$ día estaba asociado a un mayor riesgo de muerte súbita cardíaca y arritmia ventricular no fatal, sin diferencias entre grupos de edad ni sexo (Tabla 2).

La razón de odds calculada se incrementó a 54,2 (95\% IC: 4,95-592,6) al excluir del análisis a los pacientes diabéticos y a 35,8 (95\% IC: 3,68-347,5) si los excluidos fueron los pacientes afectados con enfermedades cardiovasculares. No pudo evaluarse específicamente el riesgo de arritmia ventricular (AV) debido a que no hubo pacientes expuestos a la droga entre los pocos casos de AV confirmados. Las estimaciones de los intervalos de confianza reflejan el pequeño tamaño de los subgrupos considerados. 


\section{Discusión}

Los resultados de esta revisión indican que el uso activo de domperidona oral está asociado significativamente con un incremento del riesgo de arritmia ventricular severa o muerte súbita cardíaca.

La inhibición de los canales de potasio codificados por hERG conduce a la prolongación del intervalo QT, lo que puede degenerar en arritmias ventriculares y eventualmente producir muerte súbita cardíaca ${ }^{23}$. Se ha demostrado que domperidona puede bloquear los canales hERG y por lo tanto, prolongar la repolarización cardíaca mediante la inhibición de la corriente rectificadora retardada de potasio $^{24,25}$. Al igual que cisaprida, domperidona es metabolizada por la vía de la isoenzima CYP3A4 de la familia del citocromo P450. Por lo tanto, el uso concomitante de drogas que usan esta vía puede resultar en un incremento de los niveles plasmáticos de domperidona aumentando el riesgo de toxicidad ${ }^{19,22}$.

Boyce et al, realizaron un ensayo clínico, aleatorizado, doble ciego, para estudiar la farmacocinética y los efectos de la domperidona y del ketoconazol -administrados solos o conjuntamentesobre el intervalo QT. El estudio farmacocinético reveló que ketoconazol aumenta la concentración plasmática de domperidona por inhibición del citocromo P450. Se observó prolongación del intervalo QT asociada a la administración de ambos fármacos, ya sea de manera individual o conjunta y en pacientes masculinos. La prolongación del intervalo QT fue más marcada durante la administración conjunta de domperidona y ketoconazol.

De Bruin et al, demostraron que el riesgo de paro cardíaco asociado a domperidona se incrementa con el uso concomitante de drogas que prolongan el intervalo QT y también con aquellas que inhiben la vía metabólica de la isoenzima CYP3A4 del citocromo P450.

Un hallazgo relevante de Johannes et al, en relación a la edad de los pacientes que usan domperidona fue que los mayores de 60 años tienen un incremento del riesgo de AV severa y muerte súbita cardíaca.

En la presente revisión se observaron diferencias en los valores de las razones de odds, diferencias que pueden explicarse por conjunción de varios factores que han contribuido a la heterogeneidad de los estudios incluidos impidiendo el cálculo de una medida resumen. Entre estos factores cabe mencionar la definición y/o selección de los casos, las variables utilizadas para ajustar las razones de odds y para realizar el análisis estratificado, la magnitud de la exposición al medicamento y la edad de los pacientes. La estimación del riesgo asociada a la dosis se informó sólo en uno de los estudios ${ }^{21}$ y la duración del tratamiento en ninguno de ellos.

Una limitación importante de los estudios individuales puede atribuirse a las bases de datos utilizadas. Johannes et al, manifestaron que no pudo encontrarse toda la información de interés tal como dosis prescripta, duración del tratamiento, cumplimiento de las indicaciones médicas y datos fisiológicos o de electrocardiograma que permitieran evaluar prolongación del intervalo QT. En general, los diseños de estudios epidemiológicos que usan registros electrónicos dependen críticamente no sólo de la cantidad de información registrada sino también de la validez de la misma y del valor predictivo de los códigos diagnósticos para identificar los eventos, especialmente en pacientes ambulatorios ${ }^{26}$.

\section{Conclusiones}

\section{Implicancias para la práctica clínica}

En esta revisión sistemática se encontró evidencia de que la administración oral de domperidona, principalmente en dosis mayores a $30 \mathrm{mg} /$ día, incrementa el riesgo de arritmia ventricular y muerte súbita cardíaca. En la República Argentina, la dosis recomendada en adultos para náuseas y vómitos es de 20 a $30 \mathrm{mg}$ tres o cuatro veces por día ${ }^{16,17}$; dosis que supera ampliamente el valor considerado de riesgo. Domperidona debería administrarse con precaución, especialmente en pacientes que la usan en forma concomitante con otros agentes que prolongan el intervalo QT o con inhibidores de CYP3A4. Para problemas de motilidad gastrointestinal, domperidona no puede ser considerada una alternativa terapéutica segura.

Los pacientes más susceptibles de sufrir efectos adversos cardíacos asociados a domperidona son aquellos con prolongación existente de los intervalos de conducción cardíaca, disturbios electrolíticos, desórdenes cardiovasculares, insuficiencia hepática o renal y polimedicación. Dado que los adultos mayores representan una población de alto riesgo, la prescripción de domperidona en este 
grupo etario debería considerarse potencialmente inapropiada, especialmente en pacientes ambulatorios. De no existir alternativas más seguras, se recomienda administrar la dosis más baja posible y con un monitoreo continuo de la función cardíaca mientras dure el tratamiento.

Es importante que los profesionales de la salud informen al sistema de farmacovigilancia los casos de sospecha de efectos adversos cardíacos asociados al uso de domperidona.

Se sugiere que los organismos reguladores de la calidad, eficacia y seguridad de los medicamentos controlen que los prospectos de domperidona incluyan información actualizada sobre el riesgo aquí evaluado.

\section{Sugerencias para estudios futuros}

Los síndromes dispépticos, el reflujo gastroesofágico y las náuseas y vómitos post quirúrgicos o asociados al uso de drogas citotóxicas, radioterapia o alimentos son enfermedades frecuentes y con alternativas terapéuticas muy limitadas. Es perentorio que los organismos responsables de las autorizaciones para la comercialización de los medicamentos conduzcan estudios multicéntricos en busca de evidencias acerca de la seguridad de domperidona.

Los estudios observacionales, como los de casos y controles y los estudios de cohortes constituyen una excelente herramienta para la investigación de los efectos adversos a drogas, pero muchos factores contribuyen a la heterogeneidad de los resultados impidiendo la realización de un metanálisis.

Para evaluar adecuadamente el riesgo de efectos adversos cardíacos asociados a domperidona sería conveniente organizar estudios de casos y controles que involucren a una población de gran tamaño contemplando las potenciales fuentes de sesgos y con énfasis en la dosis y en la edad del paciente. Se podría llevar a cabo mediante una investigación colaborativa que involucre a muchos centros de atención sanitaria, con directivas claras acerca de la definición del evento, identificación de los casos, verificación y selección de los mismos, descripción de la exposición y selección de los controles. Se deberá acordar cuáles son las covariables potencialmente importantes, el método para controlar los factores de confusión y la selección de los subgrupos de interés para el análisis estratificado.
Finalmente, otro elemento a tener en cuenta en trabajos futuros es la fuente de información. En los estudios de casos y controles incluidos en esta revisión se utilizaron bases de datos electrónicas con reconocidas limitaciones. Las fuentes primarias tales como las entrevistas a médicos y pacientes pueden aportar información relevante no contemplada en los sistemas automatizados vigentes y es una opción viable si el estudio es realizado con la colaboración de múltiples centros.

Agradecimientos: Los autores de la presente revisión agradecen la desinteresada colaboración de la Dra. Nora Beatriz Quaglia. En calidad de Médica y Doctora en Ciencias Biomédicas brindó asesoramiento en temas específicos y realizó una minuciosa lectura del informe final devolviendo enriquecedores comentarios.

\section{Referencias}

1. Sharkey K, Wallace J. Tratamiento de los trastornos de la motilidad intestinal y del flujo de agua; antieméticos; fármacos utilizados en las enfermedades biliares y pancreáticas. En: Goodman \& Gilman. Las Bases Farmacológicas de la Terapéutica. Editorial Mc Graw Hill $12^{\circ}$ edición 2012. p 1326.

2. Benatar A, Feenstra A, Decraene T, Vandenplas Y. Cisapride plasma levels and corrected QT interval in infants undergoing routine polysomnography. J Pediatr Gastroenterol Nutr 2001; 33: 41-6.

3. Osborne RJ, Slevin ML, Hunter RW, Hamer J. Cardiac arrhythmias during cytotoxic chemotherapy: role of domperidone. Hum Toxicol 1985; 4: 617-26.

4. Rocha CM, Barbosa MM. QT interval prolongation associated with the oral use of domperidone in an infant. Pediatr Cardiol 2005; 26: 720-3.

5. Strauss SM, Sturkenboom MC, Bleumink GS, Dieleman JP, van der Lei J, de Graeff PA, et al. Non-cardiac QTcprolonging drugs and risk of sudden cardiac death. Eur Heart J 2005; 26: 2007-12.

6. Deepak P, Ehrenpreis ED, Sadozai Y, Sifuentes H. Tu1470 Co-Prescription of Interacting Medications, Arrhythmias and Treatment With Domperidone. Gastroenterology 2012; 142 (5 Supplement 1), S-841-2.

7. Agencia Española de Medicamentos y Productos Sanitarios. Disponible en: http://www.aemps.gob.es/ [Consultado el 4 de abril de 2014].

8. Health Canada. Santé Canada. Association of domperidone maleate with serious ventricular arrhythmias and 
sudden death. Canadá. Disponible en: http://www.hc-sc. gc.ca/ [Consultado el 27 de marzo de 2014].

9. Administración Nacional de Medicamentos, Alimentos y Tecnología Médica. Disponible en: http://www.anmat. gov.ar/farmacovigilancia/ [Consultado el 4 de abril de 2014].

10. World Health Organization. Disponible en: http://www. who.int/ [Consultado el 27 de marzo de 2014].

11. The Medicines and Healthcare Products Regulatory Agency. Disponible en: http://www.mhra.gov.uk/. [Consultado el 27 de marzo de 2014].

12. Medicines Control Council. Interaction between ketoconazole and domperidone and the risk of QT prolongation - important safety information. S Afr Med J 2006; 96 (7): 596.

13. European Medicines Agency. Disponible en: http://www. ema.europa.eu/ [Consultado el 8 de abril de 2014].

14. Instituto de Salud Pública de Chile. Disponible en: http://www.ispch.cl/ [Consultado el 8 de abril de 2014].

15. Food and Drug Administration. Disponible en: http:// www.fda.gov/ [Consultado el 8 de abril de 2014].

16. Confederación Médica de la República Argentina. Medicamentos que afectan el Sistema Gastrointestinal. En: Formulario Terapéutico Nacional. Buenos Aires, Argentina: Editorial COMRA, $11^{\text {va }}$ edición. 2010. p. 247-72.

17. Comisión de Medicamentos Fundación CONAMED. Aparato Digestivo. En: Formulario Terapéutico Conamed. Buenos Aires, Argentina: Editorial Biotecnológica S.R.L, $6^{a}$ edición. 2011. p. 691-718.

18. The Cochrane Collaboration. Manual Cochrane de revisiones sistemáticas de intervenciones. Disponible en: www. cochrane.es/files/handbookcast/Manual_Cochrane_510. pdf [Consultado el 15 de marzo de 2014].

19. De Bruin M, Langendijk P, Koopmans R, Wilde A, Leufkens H, Hoes A. In-hospital cardiac arrest is asso- ciated with use of non-antiarrhythmic QTc-prolonging drugs. Br J Clin Pharmaco 2006; 63 (2): 216-23.

20. Johannes C, Varas-Lorenzo C, McQuay L, Midkiff K, Fife D. Risk of serious ventricular arrhythmia and sudden cardiac death in a cohort of users of domperidone: a nested case-control study. Pharmacoepidem Dr S 2010; 19: 881-8.

21. van Noord C, Dieleman J, van Herpen G, Verhammen K, Sturkenboom M. Domperidone and Ventricular Arrythmia or Sudden Cardiac Death. Drug Saf 2010; 33 (11): 1003-14.

22. Boyce M, Baisley K, Warrington S. Pharmacokinetic interaction between domperidone and ketoconazole leads to QT prolongation in healthy volunteers: a randomized, placebo-controlled, double-blind, crossover study. Brit J Clin Pharmacol 2011; 73 (3): 411-21.

23. Redfern WS, Carlsson L, Davis AS. Lynch WG, MacKenzie I, Palethorpe S, et al. Relationships between preclinical cardiac electrophysiology. Clinical QT interval prolongation and torsade de pointes for a broad range of drugs: evidence for a provisional safety margin in drug development. Cardiovasc Res 2003; 58: 32-45.

24. Drolet B, Rousseau G, Deleau P, Cardinal R, Turgeon J. Domperidone should not be considerd a non-risk alternative to cisapride in the treatment of gastrointestinal motility disorders. Circulation 2000; 102: 1883-5.

25. Hondeghem MD. Low safety index of domperidone: mechanism for increased odds ratio for sudden cardiac death. Acta Cardiol 2011; 66 (4): 421-5.

26. Hennessy S, Leonard CE, Palumbo CM, Bilker WB, Newcomb C, Kimmel SE. Diagnostic codes for sudden cardiac death and ventricular arrhythmia functioned poorly to identify outpatient events in EPIC's General Practice Research Database. Pharmacoepidem Dr S 2008; 17 (12): 1131-6. 